\title{
A Nonlinear Quantum Walk
}

\section{Rui FU, Meng LI}

Abstract: In this paper, we consider a nonline quantum walk. Among others, we show that the nonlinear quantum walk has the same probability distribution as its linear counterpart.

Keywords: nonlinear quantum walk, evolution process, nonlinear mapping, mathematical induction.

\section{INTRODUCTION}

The concept of quantum was first proposed by Max Planck at the beginning of the twentieth century, and has drawn great attention since then. In recent decades, quantum information theory and technology have been developed rapidly. Especially in 1993, Aharonov at al. introduced quantum walks, which are quantum analogs of classical random walks. Quantum walks can play an important role in the theory of quantum field, which is an important cornerstone of quantum information theory and technology. Quantum walks are also widely used in quantum cryptography, quantum computing and quantum algorithms.

In 2007, Navarrete-Benlloch et al. proposed a nonlinear quantum walk for the first time in [3]. The nonlinear Dirac equation is discussed in $[1,8]$ via the evolution process of a nonlinear quantum walk. Similarly, the nonlinear Schrodinger equation is also investigated in [6] with the evolution process of a nonlinear quantum walk. In [2], the weak limit theory of a nonlinear quantum walk is developed by using the scattering method and the limit distribution is obtained. In this paper, we consider a nonlinear quantum walk of the form

$$
\begin{aligned}
\Psi_{\mathrm{n}+1}(x)= & e^{-i f\left(\Psi_{n}(x+1)\right)} P_{0} \Psi_{n}(x+1)+ \\
& e^{i f\left(\Psi_{n}(x-1)\right)} Q_{0} \Psi_{n}(x-1), x \in \mathrm{Z}, n \in \bullet,
\end{aligned}
$$

Where $P_{0}, Q_{0}$ are the coin matrices and $\mathrm{f}$ is a real-valued function. Among others, we will show that the above nonlinear quantum walk has the same probability distribution as its linear counterpart.

Rui Fu, School of Mathematics and statistics, Northwest Normal University, Lanzhou, Gansu, China, Mobile No17393150135.

\section{PRELIMINARIES}

We first fix some notions and notation commonly used in this paper. As usual, $l^{2}\left(\mathrm{Z}, \mathrm{C}^{2}\right)$ represents the space of square summable $\mathrm{C}^{2}$-valued functions defined on $\mathrm{Z}$, namely

$$
l^{2}\left(\mathrm{Z}, \mathrm{C}^{2}\right)=\left\{\Phi:\left.\mathrm{Z} \rightarrow \mathrm{C}^{2}\left|\sum_{x \in Z}\right| \Phi(x)\right|^{2}<\infty\right\},
$$

Where $|\cdot|$ is the norm in $\mathrm{C}^{2}$. By convention, $M(2, \mathrm{C})$ denotes the set of two-by-two complex matrices, while $\mathrm{U}(2)$ is the set of two-by-two unitary matrices.

Lemma 2.1:[2] For $\mathrm{Q}(\mathrm{x})=\left(\begin{array}{cc}0 & 0 \\ c(x) & d(x)\end{array}\right), \mathrm{P}(\mathrm{x})=$

$\left(\begin{array}{cc}a(x) & b(x) \\ 0 & 0\end{array}\right)$, and $P(x)+Q(x) \in U(2)$. Then there exists isometric linear operator $\mathcal{A}: l^{2}\left(\mathrm{Z}, \mathrm{C}^{2}\right) \rightarrow l^{2}\left(\mathrm{Z}, \mathrm{C}^{2}\right)$ satisfys $[A \Phi](x)=P(x+1) \Phi(x+1)+Q(x-1) \Phi(x-1),(1.1)$ where $x \in \mathrm{Z}, \Phi \in l^{2}\left(\mathrm{Z}, \mathrm{C}^{2}\right)$.

Proof. Define $f_{\Phi}: \mathrm{Z} \rightarrow \mathrm{C}^{2}$ as

$f_{\Phi}=P(x+1) \Phi(x+1)+Q(x-1) \Phi(x-1), x \in \mathrm{Z} .(1.2)$ Because

$\sum_{i=1}\left|f_{\phi}(x)\right|^{2}$

$=\sum_{x \in 1}|P(x+1) \Phi(x+1)+Q(x-1) \Phi(x-1)|^{2}$

$=\sum_{-1}\langle P(x+1) \Phi(x+1)+Q(x-1) \Phi(x-1), P(x+1) \Phi(x+1)+Q(x-1) \Phi(x-1)\rangle_{\mathrm{C}^{2}}$

$=\sum_{x \in I}\langle P(x+1) \Phi(x+1), P(x+1) \Phi(x+1)\rangle_{\mathrm{C}^{2}}+\sum_{x \in \mathcal{E}}\langle P(x+1) \Phi(x+1), Q(x-1) \Phi(x-1)\rangle_{\mathrm{C}^{2}}$

$+\sum_{x \in I}\langle Q(x-1) \Phi(x-1), P(x+1) \Phi(x+1)\rangle_{\mathrm{C}^{2}}+\sum_{\mathrm{x} \in \mathbb{L}}\langle Q(x-1) \Phi(x-1), Q(x-1) \Phi(x-1)\rangle_{\mathrm{C}^{2}}$

$=\sum_{i=1}|P(x+1) \Phi(x+1)|^{2}+\sum_{i=1}<\Phi(x+1), P^{*}(x+1) Q(x-1) \Phi(x-1)>_{\mathrm{C}^{2}}$

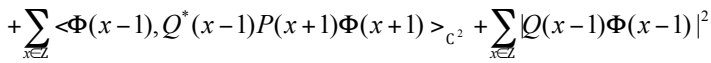

$=\sum_{x \in \mathcal{C}}|P(x) \Phi(x)|^{2}+\sum_{\mathrm{x} \in \mathrm{L}}\left\langle\Phi(x+1), P^{*}(x+1) Q(x-1) \Phi(x-1)\right\rangle_{\mathrm{C}^{2}}$

$+\sum_{x \in \mathcal{L}}<\Phi(x-1), Q^{*}(x-1) P(x+1) \Phi(x+1)>_{\mathrm{C}^{2}}+\left.\sum_{x \in Q^{\prime}} Q(x) \Phi(x)\right|^{2}$. 


\section{A Nonlinear Quantum Walk}

For $\mathrm{P}(\mathrm{x})=\left(\begin{array}{cc}a(x) & b(x) \\ 0 & 0\end{array}\right), \mathrm{Q}(\mathrm{x})=\left(\begin{array}{cc}0 & 0 \\ c(x) & d(x)\end{array}\right)$,

and $P(x)+Q(x) \in U(2)$. It is easy to get

$$
P^{*}(x+1) Q(x-1)=\left(\begin{array}{ll}
0 & 0 \\
0 & 0
\end{array}\right), Q^{*}(x-1) P(x+1)=
$$

$\left(\begin{array}{ll}0 & 0 \\ 0 & 0\end{array}\right)$. So we have

$$
\begin{aligned}
\sum_{x \in Z}\left|f_{\Phi}(x)\right|^{2} & =\sum_{x \in L}|P(x) \Phi(x)|^{2}+\sum_{x \in Z}|Q(x) \Phi(x)|^{2} \\
& =\sum_{x \in Z}|P(x) \Phi(x)+Q(x) \Phi(x)|^{2} \\
& =\sum_{x \in Z}|U(2) \Phi(x)|^{2} \\
& =\sum_{x \in Z}|\Phi(x)|^{2} .(1.3)
\end{aligned}
$$

Because $\Phi \in l^{2}\left(\mathrm{Z}, \mathrm{C}^{2}\right)$, we get $\sum_{x \in Z}\left|f_{\Phi}(x)\right|^{2}<\infty$.

Suppose $\mathcal{A} \Phi=f_{\Phi}$, and then we can get (1.1).

Furthermore,

$$
\sum_{x \in Z}|\not A \Phi(x)|^{2}=\mathrm{P} \mathscr{A} \Phi \mathrm{P}_{l^{2}\left(\mathrm{Z}, \mathrm{C}^{2}\right)}<\infty
$$

Therefore, $A \Phi \in\left(\mathrm{Z}, \mathrm{C}^{2}\right)$. According to the equation (1.3), The operator $\mathcal{A}$ is isometry. For any $\Phi^{1}, \Phi^{2} \in l^{2}\left(\mathrm{Z}, \mathrm{C}^{2}\right) . x \in \mathrm{Z}, k_{1}, k_{2} \in^{\circ}$.

$\left[A\left(k_{1} \Phi^{1}+k_{2} \Phi^{2}\right)\right](x)$

$=P(x+1)\left(k_{1} \Phi^{1}+k_{2} \Phi^{2}\right)(x+1)+Q(x-1)\left(k_{1} \Phi^{1}+k_{2} \Phi^{2}\right)(x-1)$

$=k_{1} \Phi^{1}(x+1) P(x+1)+k_{2} \Phi^{2}(x+1) P(x+1)+k_{1} \Phi^{1}(x-1) Q(x-1)+k_{2} \Phi^{2}(x-1) Q(x-1)$

$=k_{1} \Phi^{1}(x+1) P(x+1)+k_{1} \Phi^{1}(x-1) Q(x-1)+k_{2} \Phi^{2}(x+1) P(x+1)+k_{2} \Phi^{2}(x-1) Q(x-1)$

$=k_{1}\left[A \Phi^{1}\right](x)+k_{2}\left[A \Phi^{2}\right](x)$

In conclusion, the operator $A$ is isometric linear.
Proposition 2.2: When $Q_{0}=\left(\begin{array}{ll}0 & 0 \\ c & d\end{array}\right), P_{0}=$

$\left(\begin{array}{ll}a & b \\ 0 & 0\end{array}\right)$, and $P_{0}+Q_{0} \in U(2)$. Then there

exists unitary operator $A: l^{2}\left(\mathrm{Z}, \mathrm{C}^{2}\right) \rightarrow l^{2}\left(\mathrm{Z}, \mathrm{C}^{2}\right)$

satisfying

$$
[A \Phi](x)=P_{0} \Phi(x+1)+Q_{0} \Phi(x-1), x \in \mathbb{\phi} .
$$

Proof. According to lemma (2.1) and the above proof. For any $x \in \mathrm{Z}, \Phi \in l^{2}\left(\mathrm{Z}, \mathrm{C}^{2}\right)$. We have

$$
[A \Phi](x)=P_{0} \Phi(x+1)+Q_{0} \Phi(x-1) \text {. }
$$

As a result, $P_{0}+Q_{0}=\mathscr{A}$. Since we have

$P_{0}+Q_{0} \in U(2), \mathcal{A}$ is unitary operator.

Definition 2.3: $[1,2]$ The evolution equation of linear quantum walk driven by unitary operator $A$ is

$$
[A \Phi](x)=P_{0} \Phi(x+1)+Q_{0} \Phi(x-1), x \in \Phi .(1.4)
$$

Among them, the linear quantum walk has the following characteristics:

(1) $l^{2}\left(\mathrm{Z}, \mathrm{C}^{2}\right)$ is state space, and states are represented by a unit vector in $l^{2}\left(\mathrm{Z}, \mathrm{C}^{2}\right)$.

(2) states are subject to the following evolution equation

$$
\Phi_{n+1}(x)=A \Phi_{n}(x), n \in \bullet, \Phi_{n}(x) \in l^{2}\left(\mathrm{Z}, \mathrm{C}^{2}\right) .
$$

Where $\Phi_{n}(x)$ represents the state at $\mathrm{x}$ at time. In particular, $\Phi_{0}(x)$ is called initial state;

(3) The probability of finding a walker at time $\mathrm{n}$ at $\mathrm{x}$ is $\mathrm{P} \Phi_{n}(x) \mathrm{P}_{c^{2}}^{2}$.

Corollary 2.4: According to the asymmetry of the operator $\mathcal{A}$, then

$$
\mathrm{P} \Phi_{n+1} \mathrm{P}_{l^{2}\left(\mathrm{Z}, \mathrm{C}^{2}\right)}^{2}=\mathrm{P} \Phi_{n} \mathrm{P}_{l^{2}\left(\mathrm{Z}, \mathrm{C}^{2}\right)}^{2}=\mathrm{L}=\mathrm{P} \Phi_{0} \mathrm{P}_{l^{2}\left(\mathrm{Z}, \mathrm{C}^{2}\right)}^{2} .(1
$$

Especially, when $\mathrm{P} \Phi_{0} \mathrm{P}_{l^{2}\left(\mathrm{Z}, \mathrm{C}^{2}\right)}=1$, 
$\mathrm{P} \Phi_{n+1} \mathrm{P}_{l^{2}\left(\mathrm{Z}, \mathrm{C}^{2}\right)}^{2}=\mathrm{P} \Phi_{n} \mathrm{P}_{l^{2}\left(\mathrm{Z}, \mathrm{C}^{2}\right)}^{2}=\mathrm{L}=\mathrm{P} \Phi_{0} \mathrm{P}_{l^{2}\left(\mathrm{Z}, \mathrm{C}^{2}\right)}^{2}=1 . \quad \sum_{x \in \mathbb{G}} \mathrm{P} e^{-i f(\Psi(x+1))} P_{0} \Psi(x+1) \mathrm{P}_{\mathrm{C}^{2}}^{2}=\sum_{x \in \mathbb{c}} \mathrm{P} P_{0} \Psi(x+1) \mathrm{P}_{\mathrm{C}^{2}}^{2}$

\section{MAIN RESULTS}

Theorem 3.1: Let $\mathrm{f}$ be a function from $\mathrm{C}^{2}$ to ${ }^{\circ}$ $Q_{0}=\left(\begin{array}{ll}0 & 0 \\ c & d\end{array}\right) \quad$ and $\quad P_{0}=\left(\begin{array}{ll}a & b \\ 0 & 0\end{array}\right) \quad$ satisfy $P_{0}+Q \in U(2)$.Then there exists a mapping $\mathcal{A}_{f}: l^{2}\left(\mathrm{Z}, \mathrm{C}^{2}\right) \rightarrow l^{2}\left(\mathrm{Z}, \mathrm{C}^{2}\right)$ as

$$
\begin{aligned}
& {\left[\mathcal{A}_{f} \Psi\right](x)} \\
& =e^{-i f(\Psi(x+1))} P_{0} \Psi(x+1)+e^{i f(\Psi(x-1))} Q_{0} \Psi(x-1),(2.1)
\end{aligned}
$$

where $\quad x \in \mathbb{\phi}, \Psi \in l^{2}\left(\mathrm{Z}, \mathrm{C}^{2}\right) \quad, \quad$ and $\mathrm{P} \mathscr{A}_{f} \Psi \mathrm{P}=\mathrm{P} \Psi \mathrm{P}$.

proof. If $\Psi \in l^{2}\left(\mathrm{Z}, \mathrm{C}^{2}\right)$, let function $\mathrm{F}: \mathrm{Z} \rightarrow \mathrm{C}^{2}$ be

$F(x)=e^{-i f(\Psi(x+1))} P_{0} \Psi(x+1)+e^{i f(\Psi(x-1))} Q_{0} \Psi(x-1), x \in \mathbb{\phi}$.

Then, we have

$$
\begin{aligned}
& \mathrm{P} F \mathrm{P}_{l^{2}\left(\mathrm{z}, \mathrm{C}^{2}\right)}^{2} \\
& =\sum_{x \in \oplus} \mathrm{P} e^{-i f(\Psi(x+1))} P_{0} \Psi(x+1)+e^{i f(\Psi(x-1))} Q_{0} \Psi(x-1) \mathrm{P}_{\mathrm{C}^{2}}^{2} \\
& =\sum_{x \in \mathbb{A}}<e^{-i f(\Psi(x+1))} P_{0} \Psi(x+1)+e^{i f(\Psi(x-1))} Q_{0} \Psi(x-1), \\
& e^{-i f(\Psi(x+1))} P_{0} \Psi(x+1)+e^{i f(\Psi(x-1))} Q_{0} \Psi(x-1)>_{C^{2}}^{2} \\
& =\sum_{x \in \mathbb{\Phi}} \mathrm{P} e^{-i f(\Psi(x+1))} P_{0} \Psi(x+1) \mathrm{P}_{\mathrm{C}^{2}}^{2} \\
& +\sum_{x \in \mathfrak{c}}<e^{-i f(\Psi(x+1))} \Psi(x+1), e^{i f(\Psi(x-1))} P_{0}^{*} Q_{0} \Psi(x-1)>_{{ }^{2}}^{2} \\
& +\sum_{x \in \mathcal{A}}<e^{i f(\Psi(x-1))} \Psi(x-1), e^{-i f(\Psi(x+1))} Q_{0}^{*} P_{0} \Psi(x+1)>_{C^{2}}^{2} \\
& +\sum_{x \in \mathscr{\Phi}} \mathrm{P} e^{i f(\Psi(x-1))} Q_{0} \Psi(x-1) \mathrm{P}_{\mathrm{C}^{2}}^{2} .
\end{aligned}
$$

Since $Q_{0}=\left(\begin{array}{ll}0 & 0 \\ c & d\end{array}\right), \quad P_{0}=\left(\begin{array}{ll}a & b \\ 0 & 0\end{array}\right)$. It is easy to get

$$
P_{0}^{*} Q_{0}=Q_{0}^{*} P_{0}=0 .(2.3)
$$

Moreover, for any $x \in \mathbb{\Phi}$, we have

Therefore, $\sum_{x \in \mathfrak{d}} \mathrm{P} e^{i f(\Psi(x-1))} Q_{0} \Psi(x-1) \mathrm{P}_{\mathrm{C}^{2}}^{2}=\sum_{x \in \mathfrak{d}} \mathrm{P} Q_{0} \Psi(x-1) \mathrm{P}_{\mathrm{C}^{2}}^{2}$

Combine(2.3), (2.4), and (2.5), we can get $\mathrm{P} F \mathrm{P}_{l^{2}\left(\mathrm{Z}, \mathrm{C}^{2}\right)}^{2}$

$=\sum_{x \in \mathbb{q}} \mathrm{P} e^{-i f(\Psi(x+1))} P_{0} \Psi(x+1) \mathrm{P}_{\mathrm{C}^{2}}^{2}+\sum_{x \in \mathbb{A}} \mathrm{P} e^{i f(\Psi(x-1))} Q_{0} \Psi(x-1) \mathrm{P}_{\mathrm{C}^{2}}^{2}$

$=\sum_{x \in \mathbb{G}} \mathrm{P} P_{0} \Psi(x+1) \mathrm{P}_{\mathrm{C}^{2}}^{2}+\sum_{x \in \mathscr{\Phi}} \mathrm{P} Q_{0} \Psi(x-1) \mathrm{P}_{\mathrm{C}^{2}}^{2}$

$=\sum_{x \in \mathbb{G}} \mathrm{P} P_{0} \Psi(x) \mathrm{P}_{\mathrm{C}^{2}}^{2}+\sum_{x \in \mathbb{G}} \mathrm{P} Q_{0} \Psi(x) \mathrm{P}_{\mathrm{C}^{2}}^{2}$

$=\sum_{x \in \mathscr{q}} \mathrm{P} P_{0} \Psi(x)+Q_{0} \Psi(x) \mathrm{P}_{\mathrm{C}^{2}}^{2}$

$=\mathrm{P} \Psi \mathrm{P}_{l^{2}\left(\mathrm{Z}, \mathrm{C}^{2}\right)}^{2} \cdot(2.6)$
And for any $\Psi(x) \in l^{2}\left(\mathrm{Z}, \mathrm{C}^{2}\right), \quad \mathrm{P} \Psi \mathrm{P}_{l^{2}\left(\mathrm{Z}, \mathrm{C}^{2}\right)}^{2}<\infty$,

we can get

$$
\mathrm{P} F \mathrm{P}_{l^{2}\left(\mathrm{z}, \mathrm{C}^{2}\right)}^{2}<\infty .
$$

Then, $\mathrm{F}(x) \in l^{2}\left(\mathrm{Z}, \mathrm{C}^{2}\right)$. For the defined mapping

$A_{f}$, define

$$
\mathcal{A}_{f}=\mathrm{F}, \Psi \in l^{2}\left(\mathrm{Z}, \mathrm{C}^{2}\right) .
$$

Obviously,

$\mathscr{A}_{f} \Psi(x)=F(x)=e^{-i f(\Psi(x+1))} P_{0} \Psi(x+1)+e^{i f(\Psi(x-1))} Q_{0} \Psi(x-1)$, where $x \in \mathbb{\Phi}, \Psi \in l^{2}\left(\mathrm{Z}, \mathrm{C}^{2}\right)$. According to (2.6) and (2.7), we can get

$$
\mathrm{P} \mathscr{A}_{f} \Psi \mathrm{P}_{l^{2}\left(\mathrm{z}, \mathrm{C}^{2}\right)}^{2}=\mathrm{P} \Psi \mathrm{P}_{l^{2}\left(\mathrm{z}, \mathrm{C}^{2}\right)}^{2} .
$$

Theorem 3.2: The mapping $\mathcal{A}_{f}$ defined above is nonlinear, for any $\Psi \in l^{2}\left(\mathrm{Z}, \mathrm{C}^{2}\right), \mathcal{A}_{f} \Psi$ is a unitary matrix.

Proof. For any $\Psi^{1}, \Psi^{2} \in l^{2}\left(\mathrm{Z}, \mathrm{C}^{2}\right)$, 


$$
\begin{aligned}
& {\left[\mathscr{A}_{f} \Psi^{1}\right](x)+\left[\mathscr{A}_{f} \Psi^{2}\right](x)} \\
& =e^{-i f\left(\Psi^{1}(x+1)\right)} P_{0} \Psi^{1}(x+1)+e^{i f\left(\Psi^{1}(x-1)\right)} Q_{0} \Psi^{1}(x-1) \\
& +e^{-i f\left(\Psi^{2}(x+1)\right)} P_{0} \Psi^{2}(x+1)+e^{i f\left(\Psi^{2}(x-1)\right)} Q_{0} \Psi^{2}(x-1)
\end{aligned}
$$

However,

$$
\begin{aligned}
& {\left[\mathcal{A}_{f}\left(\Psi^{1}+\Psi^{2}\right)\right](x)} \\
& =e^{-i f\left(\Psi^{1}(x+1)+\Psi^{2}(x+1)\right)} P_{0} \Psi^{1}(x+1)+e^{-i f\left(\Psi^{1}(x+1)+\Psi^{2}(x+1)\right)} P_{0} \Psi^{2}(x+1) \\
& +e^{i f\left(\Psi^{1}(x-1)+\Psi^{2}(x-1)\right)} Q_{0} \Psi^{1}(x-1)+e^{i f\left(\Psi^{1}(x-1)+\Psi^{2}(x-1)\right)} Q_{0} \Psi^{2}(x-1) .(2.9)
\end{aligned}
$$

It is obvious that $(2.8) \neq(2.9)$. Then is $\mathcal{A}_{f}$ a nonlinear mapping. According to (2.1) and the definition of $Q_{0}$ and $P_{0}$, for any $\Psi \in l^{2}\left(\mathrm{Z}, \mathrm{C}^{2}\right)$

$$
\begin{aligned}
& \left(\mathscr{A}_{f} \Psi\right)^{*}\left(\mathscr{A}_{f} \Psi\right) \\
& =\left(e^{-i f(\Psi)} P_{0}+e^{i f(\Psi)} Q_{0}\right)^{*}\left(e^{-i f(\Psi)} P_{0}+e^{i f(\Psi)} Q_{0}\right) \\
& =\left(e^{i f(\Psi)} P_{0}^{*}+e^{-i f(\Psi)} Q_{0}^{*}\right)\left(e^{-i f(\Psi)} P_{0}+e^{i f(\Psi)} Q_{0}\right) \\
& =P_{0}^{*} P_{0}+e^{2 i f(\Psi)} P_{0}^{*} Q_{0}+e^{-2 i f(\Psi)} Q_{0}^{*} P_{0}+Q_{0}^{*} Q_{0} .
\end{aligned}
$$

For $P_{0}=\left(\begin{array}{ll}a & b \\ 0 & 0\end{array}\right)$, we get

$$
P_{0}^{*}=\left(\begin{array}{ll}
\bar{a} & 0 \\
\bar{b} & 0
\end{array}\right), P_{0}^{*} P_{0}=\left(\begin{array}{ll}
|a|^{2} & \bar{a} \cdot b \\
\bar{b} \cdot a & |b|^{2}
\end{array}\right)
$$

Similarly,

$$
Q_{0}^{*} Q_{0}=\left(\begin{array}{ll}
|c|^{2} & \bar{c} \cdot d \\
\bar{d} \cdot c & |d|^{2}
\end{array}\right) .
$$

Since $P_{0}+Q_{0} \in U(2)$, we get $P_{0}^{*} P_{0}+Q_{0}^{*} Q_{0}=I$. Namely,

$$
\left(\mathscr{A}_{f} \Psi\right)^{*}\left(\mathscr{A}_{f} \Psi\right)=I
$$

Similarly,

$$
\left(\mathscr{A}_{f} \Psi\right)\left(\mathscr{A}_{f} \Psi\right)^{*}=I
$$

In conclusion, $\mathscr{A}_{f} \Psi$ is a unitary matrix.

Definition 3.3: The evolution equation of nonlinear quantum walk driven by $\mathcal{A}_{f}$ is

$$
\begin{aligned}
& \mathscr{A}_{f}\left(\Psi_{n}(x)\right) \\
& =e^{-i f\left(\Psi_{n}(x+1)\right)} P_{0} \Psi_{n}(x+1)+e^{i f\left(\Psi_{n}(x-1)\right)} Q_{0} \Psi_{n}(x-1), x \in \mathbb{\Phi}, n \in \bullet .
\end{aligned}
$$

Among them, the nonlinear quantum walk has the following characteristics:

(1) $l^{2}\left(\mathrm{Z}, \mathrm{C}^{2}\right)$ is state space, and states are repre-

sented by a unit vector in $l^{2}\left(\mathrm{Z}, \mathrm{C}^{2}\right)$;

(2) states are subject to the following evolution equation

$$
\Psi_{n+1}=A \Psi_{n}, n \in \bullet
$$

In particular, $\Psi_{0}$ is the initial state;

(3) The probability of finding a walker at time $\mathrm{n}$ at $\mathrm{x}$ is $\mathrm{P \Psi}_{n}(x) \mathrm{P}_{\mathrm{C}^{2}}^{2}$.

Proposition 3.4: In the evolution of nonlinear quantum walk driven by $\mathcal{A}_{f}$, we can get

$\mathrm{P} \Psi_{n+1} \mathrm{P}_{l^{2}\left(\mathrm{Z}, \mathrm{C}^{2}\right)}=\mathrm{P} \Psi_{n} \mathrm{P}_{l^{2}\left(\mathrm{Z}, \mathrm{C}^{2}\right)}=\mathrm{L}=\mathrm{P} \Psi_{0} \mathrm{P}_{l^{2}\left(\mathrm{Z}, \mathrm{C}^{2}\right)}, n \in \bullet$.(2.10)

proof. According to theorem 2.1, there have $\mathrm{P} \mathscr{A}_{f} \Psi \mathrm{P}=\mathrm{P} \Psi \mathrm{P}$. From the nonlinear quantum walk evolution equation $\Psi_{n+1}=\mathscr{A} \Psi_{n}$, we get

$$
\mathrm{P} \Psi_{n+1} \mathrm{P}_{l^{2}\left(\mathrm{Z}, \mathrm{C}^{2}\right)}=\mathrm{P} \Psi_{n} \mathrm{P}_{l^{2}\left(\mathrm{Z}, \mathrm{C}^{2}\right)},(
$$

where $n \in \bullet$. Especially, When $\mathrm{n}=0$, we get

$$
\mathrm{P} \Psi_{1} \mathrm{P}_{l^{2}\left(\mathrm{Z}, \mathrm{C}^{2}\right)}^{2}=\mathrm{P} \Psi_{0} \mathrm{P}_{l^{2}\left(\mathrm{Z}, \mathrm{C}^{2}\right)} \text {. }
$$

To sum up, we get (2.10).

Corollary 3.5: Let function $\mu: x \rightarrow^{\circ}$, when $\mu(x)=\mathrm{P} \Psi(x) \mathrm{P}_{\mathrm{C}^{2}}^{2}, \mathrm{P} \Psi(x) \mathrm{P}_{l^{2}\left(\mathrm{Z}, \mathrm{C}^{2}\right)}=1$, then $\mu$ is a probability measure.

Theorem 3.6: When $\Psi_{0}(x)=\Phi_{0}(x), P(x)=P_{0}$, $Q(x)=Q_{0}$, The probability distribution of nonlinear quantum walk driven by $\mathscr{A}_{f}$ is the same as that of linear quantum walk driven by $A$.

Proof. When $P(x)=P_{0}, Q(x)=Q_{0}$, the linear quantum walk evolution process driven by $A$ is $\left[A \Phi_{n}\right](x)=P_{0} \Phi_{n}(x+1)+Q_{0} \Phi_{n}(x-1), x \in \mathbb{\Phi}, n \in \bullet$.(2.12)

For any $x \in \mathbb{\phi}$, it satisfies $\Psi_{0}(x)=\Phi_{0}(x)$ when $\mathrm{n}=0$. Therefore,

If $\mathrm{n}=\mathrm{k}$, we have 


\section{A Nonlinear Quantum Walk}

$$
\Psi_{k}(x)=\Phi_{k}(x), \mathrm{P}_{k}(x) \mathrm{P}_{\mathrm{C}^{2}}^{2}=\mathrm{P} \Phi_{k}(x) \mathrm{P}_{\mathrm{C}^{2}}^{2} .
$$

For $\Psi_{k+1}(x)$, we get

$$
\begin{aligned}
& \mathrm{P} \Psi_{k+1}(x) \mathrm{P}_{\mathrm{C}^{2}}^{2} \\
& =\mathrm{P} e^{-i f\left(\Psi_{k}(x+1)\right)} P_{0} \Psi_{k}(x+1)+e^{i f\left(\Psi_{k}(x-1)\right)} Q_{0} \Psi_{k}(x-1) \mathrm{P}_{\mathrm{C}^{2}}^{2} \\
& =\mathrm{P} e^{-i f\left(\Psi_{k}(x+1)\right)} P_{0} \Psi_{k}(x+1) \mathrm{P}_{\mathrm{C}^{2}}^{2}+\mathrm{P} e^{i f\left(\Psi_{k}(x-1)\right)} Q_{0} \Psi_{k}(x-1) \mathrm{P}_{\mathrm{C}^{2}}^{2} \\
& =\mathrm{P} P_{0} \Psi_{k}(x+1) \mathrm{P}_{\mathrm{C}^{2}}^{2}+\mathrm{P} Q_{0} \Psi_{k}(x-1) \mathrm{P}_{\mathrm{C}^{2}}^{2} \\
& =\mathrm{P} P_{0} \Psi_{k}(x+1)+Q_{0} \Psi_{k}(x-1) \mathrm{P}_{\mathrm{C}^{2}}^{2} \cdot(2 \cdot 13) \\
& \text { For } \Psi_{k+1}(x), \text { we have }
\end{aligned}
$$$$
\mathrm{P} \Phi_{k+1}(x) \mathrm{P}_{\mathrm{C}^{2}}^{2}
$$$$
=\mathrm{P} P_{0} \Phi_{k}(x+1)+Q_{0} \Phi_{k}(x-1) \mathrm{P}_{\mathrm{C}^{2}}^{2}
$$$$
=\mathrm{P} P_{0} \Phi_{k}(x+1) \mathrm{P}_{\mathrm{C}^{2}}^{2}+\mathrm{P} Q_{0} \Phi_{k}(x-1) \mathrm{P}_{\mathrm{C}^{2}}^{2}
$$$$
=\mathrm{P} P_{0} \Phi_{k}(x+1)+Q_{0} \Phi_{k}(x-1) \mathrm{P}_{\mathrm{C}^{2}}^{2} \cdot(2.14)
$$

When $\mathrm{n}=\mathrm{k}$, for any $x \in \mathbb{\Phi}$,

$$
\Psi_{k}(x)=\Phi_{k}(x) \text {. }
$$

Obviously,

$$
\Psi_{k}(x+1)=\Phi_{k}(x+1), \quad \Psi_{k}(x-1)=\Phi_{k}(x-1) .
$$

Combine (2.13) and (2.14), we get

$$
\begin{aligned}
& \mathrm{P} \Psi_{k+1}(x) \mathrm{P}_{\mathrm{C}^{2}}^{2}=\mathrm{P} \Phi_{k+1}(x) \mathrm{P}_{\mathrm{C}^{2}}^{2} .(2.15) \\
& \text { In conclusion, since } \mathrm{n} \text { is }
\end{aligned}
$$

$\mathrm{P}_{n}(x) \mathrm{P}_{\mathrm{C}^{2}}^{2}=\mathrm{P} \Phi_{n}(x) \mathrm{P}_{\mathrm{C}^{2}}^{2}, n \in \bullet, x \in \mathbb{Q}$.

Therefore, the probability distribution of nonlinear quantum walk driven by $\mathcal{A}_{f}$ is the same as that of linear quantum walk driven by $A$.

\section{REFERENCE}

[1] Maeda M, Suzuki A. Continuous limits of linear and nonlinear quantum walks[J]. Reviews in Mathematical Physics, 2019.

[2]Maeda M, Sasaki H, Segawa E. et al. Weak limittheorem for a nonlinear quantum walk[J]. Quantum Information Processing, 2018 , 215(17): 11128 .

[3] Navarrete-Benlloch C, et al. Nonlinear optical Galton board[J]. Physical Review A, 2007, 75(6):62333.

[4 ] Portugal R. Quantum Walks and Search Algorithms[M]. Springer New York, 2013.

[5] Venegas-Andraca S E. Quantum walks: a comprehensive review[J]. Quantum Information Processing, 2012, 11(5):1015-1106.

[6] Maeda M, Sasaki H,Segawa E, et al. Scattering and inverse scattering for nonlinear quantum walks[J]. Discrete Continuous Dynamical Systems A, 2018, 38(7):3687-3703.

[7] Konno N. Quantum random walks in one dimension[J]. Quantum Information Processing, 2002, 1(5):345-354.

[8] Lee C W, Pawe Kurzyński, Nha H. Quantum walk as a simulator of nonlinear dynamics: Nonlinear Dirac equation and solitons[J]. Physical Review A, 2015, 92(5).
[9] Hall B C. Quantum Theory for Mathematicians[M]. Springer New York, 2013.

[10] Holevo A S. Probabilistic and Statistical Aspects of Quantum Theory[M]. NORTH-HOLLAND, 1982.

[11] Ohya M, Volovich I V. Mathematical Foundation of Quantum Information and Computation[M]. 2012.

[12] Aharonov Y, Davidovich L, Zagury N. Quantum random walks[J]. Physical Review A, 1993, 48(2):1687.

Rui Fu, School of Mathematics and Statistics, Northwest Normal University, Lanzhou, China. Mobile , 86-17393150135

Meng Li, School of Mathematics and Statistics, Northwest Normal University, Lanzhou, China, 